

\title{
Vectorial characterization of DVB-T propagation channel - Application to radio-Mobile communications : the CAVITE Project
}

Christian Brousseau, Stéphane Avrillon, Franck Nivole, Louis Bertel

\section{- To cite this version:}

Christian Brousseau, Stéphane Avrillon, Franck Nivole, Louis Bertel. Vectorial characterization of DVB-T propagation channel - Application to radio-Mobile communications: the CAVITE Project. EuCAP 2007 - 2nd European Conference on Antennas and Propagation, Nov 2007, Edimbourg, United Kingdom. pp.1. hal-00322966

\section{HAL Id: hal-00322966 https://hal.science/hal-00322966}

Submitted on 19 Sep 2008

HAL is a multi-disciplinary open access archive for the deposit and dissemination of scientific research documents, whether they are published or not. The documents may come from teaching and research institutions in France or abroad, or from public or private research centers.
L'archive ouverte pluridisciplinaire HAL, est destinée au dépôt et à la diffusion de documents scientifiques de niveau recherche, publiés ou non, émanant des établissements d'enseignement et de recherche français ou étrangers, des laboratoires publics ou privés. 


\title{
VECTORIAL CHARACTERIZATION OF DVB-T PROPAGATION CHANNEL - APPLICATION TO RADIO- MOBILE COMMUNICATIONS: THE “CAVITE” PROJECT
}

\author{
C. Brousseau, S. Avrillon, F. Nivole, L. Bertel \\ Institut d'Electronique et de Télécommunications de Rennes, \\ Université de Rennes 1, France \\ Christian.Brousseau@univ-rennes1.fr
}

Keywords: Communications, DVB-T, radio-mobile, channel sounding, antenna.

\begin{abstract}
In this paper, the whole project CAVITE is described. This project is proposed within the framework of sets of themes around "Sciences and Technologies of Information and Communication" for radio-mobile digital communication in order to increase the data rate or quality of transmission. One of the objectives is the reception of Digital Video Broadcasting on Terrestrial networks (DVB-T) in vehicular condition (car, train ...).
\end{abstract}

\section{Introduction}

Several countries develop systems allowing the reception of images of television inside the vehicles. Already European manufacturers offer, at the back of the cars, DVD readers with flat-faced screens placed at the back of the front seats. In the case of DVB-T reception, quality of the receiving images depends on multiples factors and especially the effect of propagation channel, the type of used antennas and the selected channel coding.

One solution is the use of multi-antenna techniques in the receiver. These SIMO techniques studied currently make it possible to improve the spectral effectiveness of digital transmissions in environments characterized with an important number of diffusers.

In such environment, the notion of dominant propagation path is replaced by a diversity of propagation paths. For a receiving antenna array with spaced out enough elements, signals received by each antenna have a low correlation, which the receiving system can then combine to decrease the bit error rate. In these techniques, diversity approach is based only on space diversity in the transmitting and receiving antenna arrays; antennas are considered as scalar elements separated by a sufficient distance to provide a spatial decorrelation of the incoming waves.

IETR - University of Rennes 1 has decided to develop studies to improve the quality of the received images on vehicular board.
The devices considered are based on achievements of the IETR in the HF frequency band (TRILION project), but with the same objective: improvement of the quality of receiving images. These studies implement heterogeneous sensors (antennas of various types) used in diversity (space, polarization and orientation). This technique has shown its great interest, with adapted coding, to increase the transmission data rates and to improve quality of the radio links.

The objective of the CAVITE project (CAractérisation Vectorielle des canaux de propagation pour la transmission d'Images de TElévision numérique terrestre vers les mobiles) is to "transpose" these techniques used within the TRILION project, towards higher frequencies, with an application to DVB-T signal reception in vehicular condition (car, train ...).

\section{State-of-art}

Since many years, the possibilities to receive DVB-T signals in a moving vehicle or in indoor environment are studied in several European IST projects, like VALIDATE, MOTIVATE, MCP and CONFLUENT.

The VALIDATE project has helped to consolidate the future of digital terrestrial TV broadcasting by verifying the DVB-T specification in detail, by ensuring that the service planning was based on a solid technical foundation and by proposing solutions to operational problems, demonstrating the potential benefits of digital terrestrial broadcasting [12]. Some tests have been realized in mobility and have led to define the MOTIVATE project, which has investigated mobile reception of digital terrestrial TV signals in single-frequency (SFN) and multifrequency (MFN) networks [2, 8]. These works has been continued in the MCP project with a focus on mobile services [11], and, later, in the CONFLUENT project where a variety of reception tests were performed using new diversity DVB-T receivers $[3,6]$.

Furthermore, components manufacturers like LSI Logic or DiBcom have developed DVB-T demodulator chipsets, respectively L64782, DIB 3000-M and DIB 7000-M, using diversity reception. These DVB-T demodulators use two receiving antennas and the signals are combined in the digital signal processing. 
Four possibilities can be used to combine the signals from the antennas [7]:

- At the antenna outputs (beam steering approach),

- At the output of channel correction process,

- At the input of the Viterbi decoder,

- At the output of the Reed-Solomon decoder.

Usually, the MRC (Maximum Ratio Combining) algorithm is used to make a coherent summation of the different demapped signals.

These works have shown, by using more than one receiving antenna and combining the signals from the separate antennas in the digital processing, it is possible to achieve more robust reception.

Due to the used centre frequency and the frequency bandwidth, implementation of a receiving antenna on a vehicle takes on many difficulties. The antennas have many mechanical constraints and must be small, aerodynamic and low cost, and must have also good electromagnetic performances (wide frequency band, angular coverage, sensitivity ...).

In the previous studies, identical antennas have been used. They have been selected as a function of these criterions and their locations have been chosen to minimize the correlation between the received signals from each antenna [6].

\section{Background study: the TRILION project}

\subsection{Introduction}

At the University of Rennes 1, a demonstrator for the digital transmission by ionospheric radio link is actually operational in a SIMO version. This system, called TRILION (Transmission d'Images par Liaison IONosphérique), works in the HF band (3-30 MHz) and has allowed to increase in a significant way, the transmission data rate of (up to $40 \mathrm{kbits} / \mathrm{s}$ in a $9 \mathrm{kHz}$ bandwidth) with regard to the current standard $(4,8 \mathrm{kbits} / \mathrm{s})[1,10]$.

For this purpose, the receiving system is connected to a heterogeneous array of HF active antennas. An antenna vectorial signal processing is implemented on a set of colocated sensors with a diversity of antenna responses as a function of Angles Of Arrival (AoA). This antenna diversity, associated with a variation of the polarization of incoming waves as a function of arrival angles, provides an effective decorrelation of the signals got in absence of any geometrical phase shift between antennas.

The corresponding multi channel processing integrates a spatio-temporal equalization which balances the distortion of the received signal in an extended bandwidth of $9 \mathrm{kHz}$. The transmission of a QAM 16 waveform with a symbol rate of 7500 bauds and a maximum power of $700 \mathrm{~W}$ establishes a radio link with a $1300 \mathrm{~km}$ range and a $30 \mathrm{kbits} / \mathrm{s}$ data rate.
As an application, still compressed images are transmitted with a robust joint source and channel coding [10].

\subsection{Heterogeneous array of H.F. antennas}

A so-called heterogeneous array is build up with non identical sensors: active monopoles or loop antennas with different orientations. A synoptic of the array is shown in figure 1. It has been demonstrated [4] that the multi channel processing may benefit from this additional diversity as the array is sensitive to the incoming wave polarization. The advantage of using such an array is illustrated in the figure 2 where eight received signals of eight different antennas are plotted. It can be noticed that the fading doesn't appear at the same time on each channel. The reason is that the incident signals are combined with varying phases resulting of the responses of each antenna.



Figure 1 : Heterogeneous antenna array.

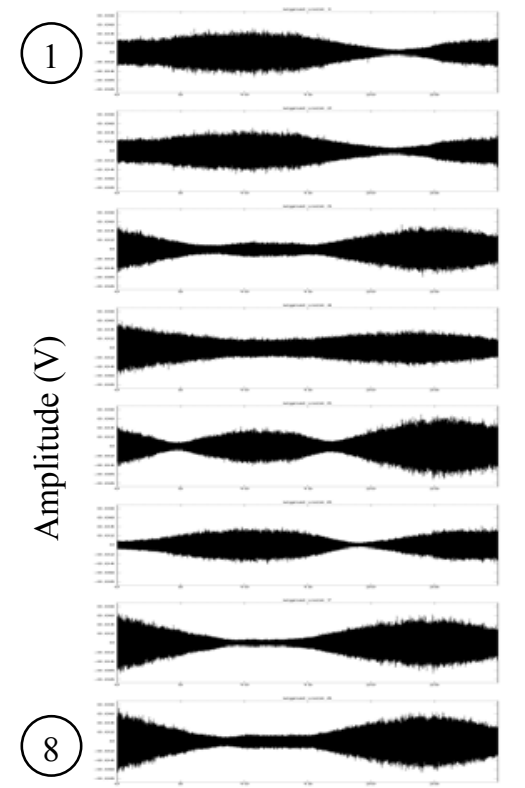

time (s)

Figure 2 : Received signals on the eight channels. 
In the application, the receiving system is set up in a limited space. A small aperture $(10 \mathrm{~m})$ array of this type is then implemented and connected to the receiver inputs. The different incident polarizations appear as decorrelation factors which balance the reduced values of the geometrical phases.

\subsection{Multichannel processing}

In the receiving system, the multichannel processing associates the following functions:

- A blind spatio temporal equalization resorting to the combination between the CMA (Constant Modulus Algorithm) and the LMS (Least Mean Square) algorithm,

- A carrier recovery, separated from the equalization, containing a second order loop filter,

- A symbol timing recovery based on a Gardner detector.

\subsection{Experimental results}

A significant $1300 \mathrm{~km}$ long link was established between France and Spain. In this experiment, the bit rate reached $30 \mathrm{kbits} / \mathrm{s}$ with a QAM 16 waveform. Many results show the efficiency of the array processing: the combination $\mathrm{CMA} \rightarrow \mathrm{LMS}$ for equalization is able to converge when fading effect or frequency selectivity occurs, and in some cases, the algorithm keeps tracking when jammers disrupt the transmission. Finally, the restored images appear to be good in terms of visual quality.

\subsection{Comments}

The originality of the technique lies in the use of different antennas (heterogeneous array). It was shown that this device could improve the quality of a digital communication by using the polarization of the incoming waves as element of decorrelation, and that the space diversity could be replaced by the diversity of the antenna responses towards the direction of arrival angles.

\section{Project description}

\subsection{Introduction}

The objective of the CAVITE project (CAracterisation Vectorielle des canaux de propagation pour la transmission d'Images de TElévision numérique terrestre vers les mobiles) is to "transpose" these techniques used within the TRILION project, towards higher frequencies, with an application to DVB-T signal reception in vehicular condition (car, train ...).

The project is divided in three parts:

- The first is a vectorial characterization of DVB-T propagation channel and the determination of the most adapted antennas. The aim of this part is to propose a vectorial channel simulator,

- The second is the study of suitable multi-antenna digital signal processing techniques, like decoding, equalization, real-time implementation, ...
And the third is to realize a demonstrator in collaboration with an industrial company.

\subsection{Objectives}

One limitation of transmission data rate on wireless communications is the coherence bandwidth which is related to frequential and temporal fadings due to the multipaths. The use of multi-antenna techniques, such as MIMO (Multiple Input - Multiple Output) techniques, allows increasing the digital data rate, thanks to the transmission and the reception of uncorrelated waves on several antennas.

In these techniques, diversity approach is based only on space diversity in the transmitting and receiving antenna arrays. Antennas are considered as scalar elements separated by a sufficient distance to provide a spatial decorrelation of the incoming waves. The whole complexity of the propagation channel is not taken into account. Several phenomenons are ignored, like:

- The waves depolarisation through the propagation channel,

- The antenna responses as a function of the type of antenna and of the arrival angles.

The studies currently undertaken around MIMO techniques consider that the antennas used are scalar sensors. Actually, the antennas cannot be regarded as scalar sensors but as complex quantity with a diversity of phase and amplitude as a function of angles of arrival, associated to an antenna vectorial signal processing.

The objectives of the project is to improve the quality of DVB-T receiving images in mobile configuration like cars, trains ... Several important points have or will be studied:

- Conception of different antennas, smallest than the wavelength. These heterogeneous antennas must have particular characteristics in terms of response in phase and magnitude. The problems of decoupling between antennas will be also studied as well as the possibility of their easy installation on a vehicle,

- Channel characterization as a function of the antenna diversity by including space diversity, in order to deduce a vectorial channel propagation model. This one must be taken into account the antenna effect (space, polarization, type),

- Development of a vectorial channel simulator to quantify the performances of various operational systems.

The originality of the technique lies in the use of different antennas (heterogeneous array). It was shown that this device could improve the quality of a digital communication by using the polarization of the incoming waves as element of decorrelation and that the space diversity could be replaced by the diversity of the antenna responses towards the direction of arrival angles. 


\subsection{Overview of project advancement}

At this time, a passive mobile channel sounder using antenna diversity has been developed [9]. This sounder uses broadcasted DVB-T received signals to measure channel propagation characteristics. The receiver is set up to receive signals from the "Rennes-Saint Pern" TDF (Télédiffusion De France) transmitter in Brittany in an $8 \mathrm{MHz}$ wide UHF channel, at the centre frequency of $650 \mathrm{MHz}$. The signals are transmitted in the $8 \mathrm{k}$ FFT mode, with a guard interval fraction of 1/32, a 64-QAM constellation and a FEC code rate of $2 / 3$ [5].

Many antennas have been developed and tested:

- Big wheel antenna,

- Patch and patch array,

- Wideband dipole,

- Pie-shaped antenna,

- Halo and printed halo antenna array,

- Loop antenna.

For the measurements, a car was equipped with the 4 inputs diversity receiver and the antennas are mounted on the roof of the vehicle. Figure 3 shows the passive diversity channel sounder and figure 4 , the embedded mobile system inside the car.



Figure 3 : Photo of the equipment used in the mobile tests.

Baseband signal processing has been developed. It includes:

- OFDM coarse-synchronization,

- Phase offset correction,

- Fine synchronization,

- Static pilot recovery and scattered pilot recovery,

- Signal equalization.

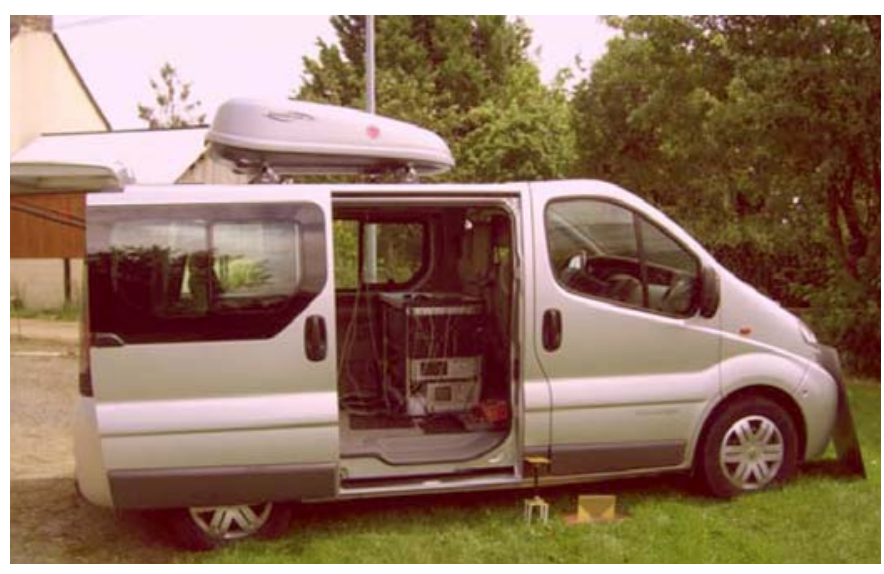

Figure 4: Photo of the embedded mobile system inside the car.

Many propagation parameters are estimated, like:

- QAM constellation,

- Channel impulse response,

- Signal to noise ratio (SNR) as a function of symbol,

- Direction of arrival angle in azimuth,

- Time - frequency channel representation.

An example of obtained results is presented in figure 5. More details and original technical parts are described in another presentation at EUCAP2007 conference [9].

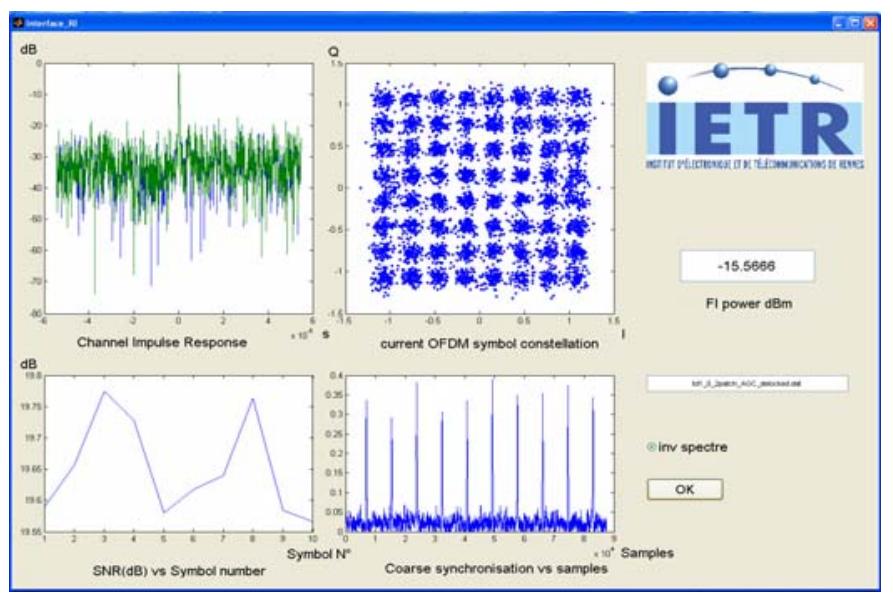

Figure 4: Example of SNR, constellation and channel estimations.

\section{Conclusion}

The CAVITE project is proposed within the framework of sets of themes around "Information Society Technologies" for radio-mobile digital communication in order to increase the data rate or quality of transmission. One of the objectives is the reception of Digital Video Broadcasting on Terrestrial networks (DVB-T) in vehicular condition (car, train ...). 
New antenna arrays, based on heterogeneous sensors, will be proposed. These antennas could be co-located and must have different characteristics in phase and amplitude, to provide a sufficient decorrelation between the received signals. These architectures will be defined from a propagation channel characterization using antenna diversity.

\section{Acknowledgements}

The authors thank the Council of Brittany - France (Conseil Régional de Bretagne) and the FNADT (Fonds National d'Aménagement et de Développement du Territoire) for their supports to this project.

\section{References}

[1] A. Bisiaux, L. Bertel, C. Barratt, D. Lemur, "Trilion project: transmission of images through the ionosphere", Eight IEE International Conference on H.F. Radio Systems and Techniques, UK, July 2000.

[2] P. Christ, P. Pogrzeba, "Introducing mobile multimedia broadcasting services", ECMAST'99 - European Conference on Multimedia Applications, Services and Techniques, Madrid, Spain, May 1999.

[3] CONFLUENT, "Report on enhancements to receiver architecture and the limits of a real demodulator and processor chip in a portable and mobile environment", IST-2001-38402, CEC Deliverable number D2.3/Task 2.3/ part 1, November 2003.

[4] Y. Erhel, D. Lemur, L. Bertel, F. Marie, "H.F. radio direction finding operating on a heterogeneous array: principles and experimental validation“, Radio-Science, vol. $39, n^{\circ} 1,2004$.

[5] ETSI, "Digital Video Broadcasting system for television, sound and data services: Draft pr ETS 300 744”, March 1997.

[6] A. Guena, D. Zapparata, A. Sibille, G. Pousset, "Mobile diversity reception of DVB-T signals using roof or windows antennas", COST 273 "Towards Mobile Broadband Multimedia Networks", $9^{\text {th }}$ Management Committee Meeting, Athens, Greece, January 2004.

[7] J.D. Mitchell, J.A. Green, "Diversity Reception of Digital Terrestrial Television (DVB-T)”, BBC R\&D White Paper, WHP 058, British Broadcasting Corporation, April 2003.

[8] MOTIVATE, "Reference receiver model for mobile DVB-T', Deliverable 06, June 2000.

[9] F. Nivole, C. Brousseau, S. Avrillon, D. Lemur, F. Marie, L. Bertel, "Comparison of antennas performances for COFDM DVB-T system - Application to channel sounding", EUCAP2007, European Conference on Antennas and Propagation, Edinburgh, UK, November 2007.

[10] C. Perrine, Y. Erhel, D. Lemur, A. Bourdillon, N. Melida, "1300 km HF radio link with a $30 \mathrm{kbits} / \mathrm{s}$ data transfer rate", Tenth IET Conference on Ionospheric Radio Systems and Techniques, London, UK, July 2006.
[11] U. Schiek, F. Klinkenberg, "The MCP terminal: A future platform for mobile services", MMC2001, Workshop on Multiradio Multimedia Communications, Berlin, Germany, November 2001.

[12] VALIDATE, "Project Final Report - WP6", AC-106, CEC Deliverable number AC106/BBC/DR/021/P/a1, June 1998. 